\title{
A NOTE ON NEAR-OPTIMAL COLORING OF SHIFT HYPERGRAPHS
}

\author{
DAVID G. HARRIS ${ }^{1}$ AND ARAVIND SRINIVASAN ${ }^{2}$
}

\begin{abstract}
As shown in the original work on the Lovász Local Lemma due to Erdős \& Lovász (Infinite and Finite Sets, 1975), a basic application of the Local Lemma answers an infinitary coloring question of Strauss, showing that given any integer set $S$, the integers may be $k$-colored so that $S$ and all its translates meet every color. The quantitative bounds here were improved by Alon, Kriz \& Nešetřil (Studia Scientiarum Mathematicarum Hungarica, 1995). We obtain an asymptotically optimal bound in this note, using the technique of iteratively applying the Lovász Local Lemma in order to prune dependencies.
\end{abstract}

\section{INTRODUCTION}

One of the first applications of the Lovász Local Lemma (LLL) 2] is in fact an affirmative answer to an infinitary question of Strauss: for a given $k$, does there exist a finite $m$ such that for any set $S$ of $m$ integers, there is a $k$-coloring of the integers such that every integer translate of $S$ (i.e., sets of the form $S+t$, for $t \in \mathbf{Z})$ meets every color class? We let $m(k)$ denote the smallest such value of $m$, if it exists.

By combining the LLL with a compactness argument, it was shown in $[2$ that $m(k) \leq(3+$ $o(1)) k \ln k$. Following this, the work of [1] showed, among other things, that $m(k) \geq(1-o(1)) k \ln k$, and also presented an "efficient" version of the upper bound, by showing that the required coloring can in fact be made periodic with a short period. Answering one of the main open questions of [1, we prove in this short note that $m(k) \leq(1+o(1)) k \ln k$ (ours is also an efficiently-computable periodic coloring as in [1]). Our approach is very similar to that of [3], and is based on the wellknown iterated LLL technique; see [4] for several applications of this technique. We also hope that a simple approach such as ours can have pedagogical use in teaching the LLL: that a simple "slowing down" in applying the LLL, can in many cases do better than a direct LLL application.

We follow the approach of [1] and reduce the problem to a certain hypergraph-coloring problem: how small an $m=m(k)$ can we exhibit, so that for every $m$-uniform, $m$-regular hypergraph $H$ there exists a $k$-coloring of the vertices such that every edge meets every color class? (Briefly, each vertex corresponds to an integer; every edge corresponds to a translation of $S$.) Thus, we use this hypergraph-coloring terminology from now on. A short calculation using the LLL - specifically, its "symmetric" special case, Theorem 1.2 - shows that if $m=(3+o(1)) k \ln k$, then there is a positive probability that a random coloring causes every edge to meet every color class [1.

Theorem 1.1. Suppose $m \geq(1+\epsilon(k)) \cdot k \ln k$ where $\epsilon(k)=(4+v(k)) \ln ^{-1 / 2} k$, and suppose $k$ is sufficiently large; $v(k)$ is a positive function of $k$ that goes to zero as $k$ increases. Then, the vertices of any m-uniform, $m$-regular hypergraph can be colored using $k$ colors, such that each edge meets every color class. Furthermore, such a coloring can be found in randomized polynomial time.

We assume that $k$ is sufficiently large. We ignore all rounding effects; in this vein, we suppose $m=(1+\epsilon) k \ln k$ exactly.

\footnotetext{
${ }^{1}$ Department of Applied Mathematics, University of Maryland, College Park, MD 20742. Research supported in part by NSF Award CNS-1010789. Email: davidgharris29@hotmail.com.

${ }^{2}$ Department of Computer Science and Institute for Advanced Computer Studies, University of Maryland, College Park, MD 20742. Research supported in part by NSF Award CNS-1010789. Email: srin@cs.umd.edu.
} 
Our proof will make two applications of the LLL. To make this note self-contained, we state a simplified version of the LLL; much greater generality is possible but will not be needed here.

Theorem 1.2 (Lovász Local Lemma; simplified form). Suppose there is a probability space $\Omega$, with events $B_{1}, \ldots, B_{l}$. (These are referred to as "bad" events.) Suppose for that for all $i=1, \ldots, l$ the following conditions hold:

(1) $P_{\Omega}\left(B_{i}\right) \leq p$

(2) The event $B_{i}$ is independent of all but d other bad-events $B_{j_{1}}, \ldots, B_{j_{d}}$;

(3) $e p(d+1) \leq 1$, where $e=2.718 \ldots$ is Euler's number.

Then, with positive probability, none of the events $B_{1}, \ldots, B_{m}$ occur.

(The definition of "dependence" in the context of the LLL is natural but slightly complicated; when the probability space $\Omega$ is derived by selecting variables independently, as it does for this note, then a sufficient condition for $B, B^{\prime}$ to be independent is that they are determined by disjoint sets of variables.)

1.1. Phase I. In Phase I, we choose a coloring using $k^{\prime}=k / \ln k$ colors; each vertex receives each color uniformly at random and independently. On average, each edge $f$ receives each color an average of $\mu=(1+\epsilon) \ln ^{2} k$ times.

For each edge $f$ and each color $c$, we have a bad-event "either $f$ receives the color more than $m_{1}=\mu(1+\delta)$ times, or less than $m_{0}=\mu(1-\delta)$ times", where $\delta=4 / \sqrt{\ln k}$. For $k$ sufficiently large, we have $\delta<1$ and the probability of this event can be estimated by the Chernoff bound; it is at most $p \leq 2 e^{-\mu \delta^{2} / 3} \leq 2 k^{-16(1+\epsilon) / 3}$. Similarly, each bad-event $(c, f)$ depends on other bad-events $\left(c^{\prime}, f^{\prime}\right)$ iff the edges $f, f^{\prime}$ intersect; hence the dependency of a bad-event is at most $d \leq k^{\prime} \times m \times m \leq(1+\epsilon)^{2} k^{3} \ln k$. For $k$ sufficiently large the LLL criterion is

$$
e \times 2 k^{-16 / 3(1+\epsilon)} \times\left(k^{3} \ln k(1+\epsilon)^{2}+1\right) \leq 1 ;
$$

this clearly holds when $k$ is sufficiently large. Note that in this phase, we are not taking advantage of the " $\epsilon$-slack" in our estimate for $m$, i.e. that $m$ is somewhat larger than $k \ln k$. That slack will not be used until Phase II.

1.2. Phase II. In the second phase of the construction, fix a good coloring as guaranteed by Phase I, and subdivide each of the initial colors from Phase I into $\ln k$ sub-colors randomly (i.e., if a vertex $u$ received color $a$ in Phase $\mathrm{I}$, its new color is $(a, b)$, where $b$ is chosen uniformly at random and independently from $\{1,2, \ldots, \ln k\})$. The total number of colors thus produced is $k^{\prime} \times \ln k=k$ as desired. The critical property here is that distinct colors from Phase I no longer affect each other in any way. This greatly reduces the dependency when applying the Lovász Local Lemma.

Now consider an edge $f$ and a color $c$ (the color $c$ includes both the coloring from Phase I and Phase II): a bad event is that $f$ does not see the color $c$. The probability of this event can be computed as follows. The edge sees the Phase-I color corresponding to $c$ at least $m_{0}$ times; hence, the probability that none of the appearances is equal to $c$, is at most $p \leq\left(1-k^{\prime} / k\right)^{m_{0}}$.

Next, consider the dependency of any fixed event $(c, f)$. Again, event $c, f$ affects $c^{\prime}, f^{\prime}$ iff $c, c^{\prime}$ have a common Phase-I color and $f, f^{\prime}$ intersect in some vertex which shares this Phase-I color. As each Phase-I color appears at most $m_{1}$ times in $f$, the total dependency is thus at most $d \leq\left(k / k^{\prime}\right) m_{1} m$. (The term $k / k^{\prime}$ here accounts for the number of choices for the Phase-II color of $c^{\prime}$.)

The LLL criterion is thus satisfied if $e\left(1-k^{\prime} / k\right)^{m_{0}}\left(\left(k / k^{\prime}\right) m_{1} m+1\right) \leq 1$. Routine calculations show that this is satisfied for $k$ sufficiently large if $\epsilon \geq(4+v) \ln ^{-1 / 2} k$. We use the standard inequality $\left(1-k^{\prime} / k\right)^{m_{0}} \leq e^{-k^{\prime} m_{0} / k}$ here; the exponent here is what requires that $\epsilon \cdot \sqrt{\ln k}$ should be slightly larger than 4 .

The bad-events in both Phase I and Phase II are easy to check, and the probability spaces are determined by independent variables, so the Moser-Tardos algorithm [5] can be employed to construct such a coloring in polynomial time. 


\section{AcKnowledgments}

We thank the referees for their helpful comments and suggestions.

\section{REFERENCES}

[1] N. Alon, I. Kriz, and J. Nešetřil. How to color shift hypergraphs. Studia Scientiarum Mathematicarum Hungarica, 30:1-12, 1995. Also in Combinatorics and its Applications to Regularity and Irregularity of Structures (W. A. Deuber and V. T. Sós, eds.), Akadémiai Kiadó, Budapest, pages 1-11, 1995.

[2] P. Erdős and L. Lovász. Problems and results on 3-chromatic hypergraphs and some related questions. In Infinite and Finite Sets, volume 11 of Colloq. Math. Soc. J. Bolyai, pages 609-627. North-Holland, 1975.

[3] U. Feige, M. M. Halldórsson, G. Kortsarz, and A. Srinivasan. Approximating the domatic number. SIAM Journal on Computing, 32:172-195, 2002.

[4] M. Molloy and B. Reed. Graph Colouring and the Probabilistic Method. Springer-Verlag, 2001.

[5] Robin Moser and Gabor Tardos. A constructive proof of the general Lovász Local Lemma. Journal of the ACM, 57(2), 2010. 